\title{
Mitochondrial DNA mutations in patients with postlingual, nonsyndromic hearing impairment
}

Howard T Jacobs*1,2 ${ }^{\star}$, Timothy P Hutchin ${ }^{3,4}$, Timo Käppi ${ }^{1}$, Greta Gillies ${ }^{1}$, Kia Minkkinen ${ }^{1}$, John Walker ${ }^{1}$, Karen Thompson ${ }^{3}$, Anja T Rovio ${ }^{1}$, Massimo Carella ${ }^{5}$, Salvatore Melchionda ${ }^{5}$, Leopoldo Zelante ${ }^{5}$, Paolo Gasparini ${ }^{6}$, Ilmari Pyykkö ${ }^{7}$, Zahid H Shah ${ }^{1}$, Massimo Zeviani ${ }^{8}$ and Robert F Mueller ${ }^{3}$

\begin{abstract}
${ }^{1}$ Institute of Medical Technology \& Tampere University Hospital, Tampere, Finland; ${ }^{2}$ IBLS Division of Molecular Genetics, University of Glasgow, UK; ${ }^{3}$ Molecular Medicine Unit, University of Leeds, UK; ${ }^{4}$ Clinical Chemistry, Birmingham Children's Hospital, Birmingham, UK; ${ }^{5}$ Medical Genetics Service, IRCCS Casa Sollievo della Sofferenza, San Giovanni Rotondo, Italy; ${ }^{6}$ Telethon Institute of Genetics and Medicine, Naples, Italy; ${ }^{7}$ Department of Otolaryngology, Tampere University Hospital, Tampere, Finland; ${ }^{8}$ National Neurological Institute 'Carlo Besta', Milan, Italy
\end{abstract}

Mitochondrial mutations have previously been reported anecdotally in families with maternally inherited, nonsyndromic hearing impairment. To ascertain the contribution of mitochondrial mutations to postlingual but early-onset, nonsyndromic hearing impairment, we screened patients collected from within two different populations (southern Italy and UK) for previously reported mtDNA mutations associated with hearing disorders. Primer extension (SNP analysis) was used to screen for specific mutations, revealing cases of heteroplasmy and its extent. The most frequently implicated tRNA genes, Leu(UUR) and Ser(UCN), were also sequenced in all Italian patients. All tRNA genes were sequenced in those UK patients showing the clearest likelihood of maternal inheritance. Causative mtDNA mutations were found in approximately $5 \%$ of patients in both populations, representing almost $10 \%$ of cases that were clearly familial. Age of onset, where known, was generally before adulthood, and hearing loss was typically progressive. Haplogroup analysis revealed a possible excess of haplogroup cluster HV in the patients, compared with population controls, but of borderline statistical significance. In contrast, we did not find any of the previously reported mtDNA mutations, nor a significant deviation from haplogroup cluster frequencies typical of the control population, in patients with late adult-onset hearing loss (age-related hearing impairment) from the UK or Finland.

European Journal of Human Genetics (2005) 13, 26-33. doi:10.1038/sj.ejhg.5201250

Published online 4 August 2004

Keywords: deafness; mitochondrial disease; transfer RNA; ribosomal RNA; aminoglycoside; haplogroup

Introduction

Many previous reports in the literature have identified maternal pedigrees with mitochondrial DNA mutations, associated with a phenotype of isolated sensorineural

*Correspondence: Dr HT Jacobs, Institute of Medical Technology, FI-33014 University of Tampere, Finland. Tel: + 35832157731; Fax: +35832157710; E-mail: howard.t.jacobs@uta.fi

Received 24 February 2004; revised 18 May 2004; accepted 25 May 2004 hearing impairment (nonsyndromic deafness) among most or all affected individuals in the family. The most commonly reported such mutations are $\mathrm{A} 1555 \mathrm{G},{ }^{1}$ A7445G, ${ }^{2,3} 7472$ insC, ${ }^{4,5}$ as well as $A 3243 \mathrm{G}^{6,7}$ which is also found in families with more severe, syndromic disease. Assignment of these mutations as pathological is based upon their absence from unaffected families, their ability to produce a biochemical phenotype in cultured cell models such as rho-zero cybrids and demonstrable effects 
on mitochondrial protein synthesis. ${ }^{8-13}$ A number of other mutations in $\mathrm{tRNA}^{\mathrm{Ser}(\mathrm{UCN})}$, that is, $\mathrm{T} 7510 \mathrm{C},{ }^{14,15}$ T7511 $\mathrm{C}^{16,17}$ and $\mathrm{T} 7512 \mathrm{C}$, $^{18}$ as well as one other mutation in 12S rRNA (C1494T) ${ }^{19}$ have been reported in cases of similar phenotypes (syndromic hearing impairment in the case of T7512C). The latter tRNA, as well as tRNA ${ }^{\mathrm{Leu}(\mathrm{UUR})}$, may be hotspots for such mutations, although other tRNAs have not been systematically excluded.

Other studies have attempted to evaluate the contribution of these mutations to hearing disorders more generally, by screening defined groups of patients for one or more of them. However, most such studies are difficult to interpret, either because the patient selection criteria were too imprecise, or alternatively too narrow (eg focusing only on families with probable maternal inheritance of hearing impairment), or else only a subset of the above mutations was investigated. In addition, virtually all previous studies have been conducted in localized populations so that their wider relevance is unclear. Although the contribution of mtDNA mutations to congenital (prelingual, early childhood onset) deafness is minor, ${ }^{20,21}$ mitochondrial involvement in the much larger group of patients with postlingual hearing impairment, that is, with onset during childhood or early adulthood, ${ }^{22,23}$ whether familial or sporadic, has not been studied systematically.

Modern populations can be stratified according to their membership of ancient matrilineal clans defined by founder polymorphisms in mtDNA. These haplogroups and their deepest branches (haplogroup clusters) show subtle differences between populations, ${ }^{24}$ for example, east-west and north-south clines within Europe (Western Eurasia). Previous authors have reported associations between specific haplogroups or haplogroup clusters and a variety of disorders, including male infertility, ${ }^{25}$ Parkinson's disease, ${ }^{26}$ Alzheimer's and other types of dementia $^{27,28}$ and multiple sclerosis, ${ }^{29}$ as well as with longevity in different populations. ${ }^{30,31}$ Not all these studies are congruent, however, and virtually no evidence is available to support a specific functional role in pathogenesis of polymorphisms characteristic of, or unique to, specific haplogroup backgrounds.

In the present study, we have analysed groups of patients with postlingual, nonsyndromic hearing impairment from different European populations, for evidence of mtDNA involvement in their disorder, looking both at known pathogenic mutations and haplogroup affiliations. For comparison, we also studied Finnish patients with agerelated hearing impairment (ARHI).

\section{Materials and methods Patients and controls}

Patients with nonsyndromic hearing impairment were recruited to the study via advertising in the newsletter of a patient organization (UK), via referrals from collaborating clinicians (Italy), or upon first attendance at an ENT clinic for fitting a hearing aid (Finland). Criteria for patient selection and other kinds of information obtained from questionnaires, clinical examination, clinical records and self-report are indicated, where relevant, under Results. All work with patients was conducted with informed consent, and with approval of the local Ethical Committee (Pirkanmaa Health District, Finland, decision R02138 of 5.11.02). UK controls were, like the patients, Caucasian adults, being mainly parents of cystic fibrosis sufferers, whose hearing was not specifically investigated. Italian controls were blood donors collected from the same geographical region as the patients.

\section{DNA extraction}

DNAs were extracted from blood or hair samples using standard methods. Blood samples collected in Finland were anonymously coded, then aliquoted and archived at $-80^{\circ} \mathrm{C}$. DNA was extracted from $200 \mu \mathrm{l}$ aliquots of frozen blood using the Nucleospin96 DNA extraction kit (Macherey-Nagel) on a Tecan Genesis RP 100 pipetting robot, under the manufacturer's recommended conditions.

\section{PCR and analytical procedures}

Segments of mitochondrial DNA of 200-1100 bp were amplified using custom primers, gel purified where necessary, and analysed by direct sequencing or primer extension (SNP analysis), using additional, appropriate primers. The $\mathrm{tRNA}^{\mathrm{Ser}(\mathrm{UCN})}$ and $\mathrm{tRNA}{ }^{\mathrm{Leu}(\mathrm{UUR})}$ regions were amplified, respectively, using primer pairs FR31/FR32 ${ }^{2}$ and FR6/FR7, ${ }^{9}$ under conditions stated in the original references. Heteroplasmy for 7472insC was assessed by primer extension (SNP analysis) as previously, ${ }^{13}$ using calibration standards constructed from mixtures of PCR products. The presence of $\mathrm{A} 1555 \mathrm{G}$ was determined by primer extension (SNP analysis) using primer 1555_GENO (5' CCCCTACGCATtTATATAGAGGAG $3^{\prime}$ ) and the ABI Prism SNaPshot ddNTP Primer Extension Kit (Applied Biosystems) under the manufacturer's recommended conditions, with a template fragment amplified from sample DNA using primer pair ok-1016-2 (5' TAGACTACGAAAGTGGCTTTAAC $3^{\prime}$ ) and mt-1601 (5' GTTCGTCCAAGTGCACTTTCCAG $3^{\prime}$ ), with annealing temperature $56^{\circ} \mathrm{C}$ and extension time $2 \mathrm{~min}$. Details of all other primer sequences and amplification, sequencing or minisequencing conditions are available on request. Haplogroups and haplogroup clusters were assigned based on the scheme of Macaulay and Richards ${ }^{24,32,33}$ (see www.stats.gla.ac.uk/ vincent/ images/skeleton07-08-02.jpg), except that np 73 was not used as an informative site for assigning membership of haplogroup cluster HV. For further details, see legend to Figure 1. 


\section{Statistical analyses}

The significance of differences in haplogroup cluster frequencies between patients and controls was assessed by $\chi^{2}$ analysis.

\section{Results}

Patient collection, inclusion criteria and clinical data In order to evaluate the contributions of mtDNA mutations to postlingual hearing impairment, we collected patients with nonsyndromic hearing impairment from within two geographically distinct European populations, southern Italy and UK. Slightly different procedures were employed, as considered appropriate to the two populations: in the UK, recruitment of individuals with progressive, familial hearing impairment, with onset in late childhood or in adulthood, was partly via the newsletter of a patient organization (RNID), and partly through referrals to the centre where the study was initiated (Department of Clinical Genetics, St James University Hospital, Leeds). Among the UK cases, $70 \%$ had a strong family history (affected first-degree relatives). In southern Italy, recruitment of patients was via clinicians. Apart from the fact that all patients had postlingual hearing impairment, there were no specific inclusion criteria applied to the Italian patients, although $40 \%$ of cases were familial. All patients were prescreened for connexin-26 mutations (GJB2 35delG, in some cases the entire GJB2 coding region) and were found to be negative.

For the UK patients, questionnaire responses and medical records allowed us to ascertain details such as the age of onset and nature of hearing impairment, although in some cases only anecdotal information on distant relatives, particularly of older generations, could be obtained. The average age of onset of hearing loss was spread across all ages (data not shown). Almost all subjects reported their hearing loss to be bilateral and progressive, which was confirmed from medical records where available. Audiograms were obtained for over half of the subjects, most of which were downsloping, that is, with the higher frequencies most affected. Of those with deaf parents plus other affected relatives, there was a marked preponderance of uniquely maternal over uniquely paternal family history (41:20 families), strongly suggesting a mitochondrial aetiological component.

Genetic analysis was finally conducted on blood DNAs from 80 individuals from different UK families, 10 of whom were classed as sporadic cases. Among the Italian patients, mutation data were obtained from blood DNAs of 165 unrelated patients, but due to the small quantities of DNA available and poor quality in some cases, not all analyses were successful for every sample.

\section{Causative mtDNA mutations in UK patients}

We screened all 80 individuals from the UK sample for the previously reported mtDNA mutations 961delT, A1555G,

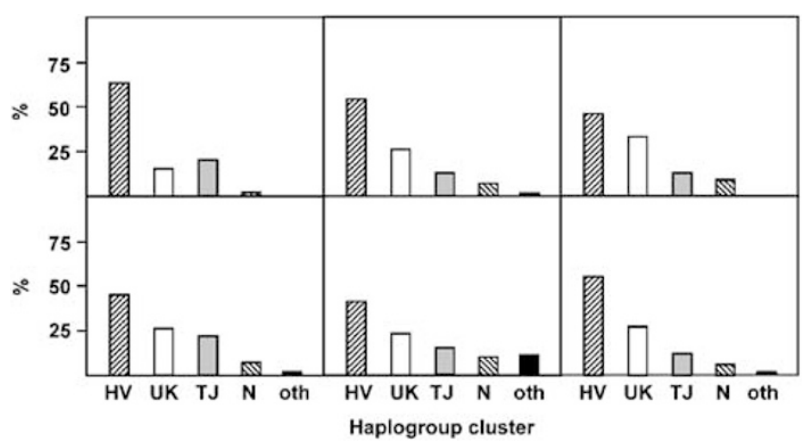

Figure 1 Frequencies of major mtDNA haplogroup clusters in different groups of patients with nonsyndromic, sensorineural hearing impairment, compared with appropriate population controls. Haplogroups and haplogroup clusters were defined essentially according to the scheme of Macaulay and Richards. ${ }^{24,32,33}$ Haplogroup cluster HV includes those in rare $\mathrm{HV}$ or pre-HV haplogroups with 11719G. Haplogroup cluster UK includes all those with $12308 \mathrm{G}$ and no other detected polymorphisms diagnostic for another haplogroup outside cluster UK. Haplogroup cluster TJ includes all those with 16126C and either 16069T or $16294 \mathrm{~T}$, but no other detected polymorphisms diagnostic for another haplogroup outside cluster TJ. Haplogroup cluster $\mathrm{N}$ includes all those with 16223T, 12705T or both, plus haplogroup-specific polymorphisms indicative of haplogroups N1a, N1b, I, W, X or $\mathrm{Y}$ (no representatives of haplogroups A or N9, which also belong to this cluster, were detected) and no other detected polymorphisms diagnostic for another haplogroup outside cluster N. 'Other' represents all other haplogroups, comprising the African/East Asian supercluster L3* defined by $10873 \mathrm{C}$, plus any assigned to haplogroups $B$ and $F$. The small number of samples $(<2 \%)$ giving conflicting data incompatible with the scheme of Macaulay and Richards were discarded from the analysis. Most likely these represent cases belonging to the major clusters, but with rare or private mutations or back-mutations at sites normally considered diagnostic. Population controls were from this study (UK), or as reported earlier by Niemi et a ${ }^{31}$ for Finnish adult controls from Tampere region (haplogroup frequencies in the same samples were verified by us using the above scheme), or De Benedictis et $a l^{30}$ for southern Italy. For statistical analysis, the frequency of haplogroup cluster HV in the Italian sample was adjusted upwards to $45 \%$ according to our own analysis of 182 independent population controls at np 11719, and the proportion of 'other' adjusted downwards to $7 \%$ in compensation. Representative of these rare haplogroups from the HV cluster accounted, similarly, for 5\% (6/123) of the southern Italian patients with postlingual hearing impairment.

A3243G, A7445G, 7472insC, T7510C, T7511C and T7512C. In addition, all 22 tRNAs were sequenced from the 15 cases most clearly showing an inheritance pattern consistent with a mitochondrial aetiology. Six cases of previously reported mutations were revealed (Table 1), comprising five among the 41 families showing uniquely 
maternal family history (two cases of A1555G, one each of T7510C, A7445G and A3243G), plus one case of 7472insC ( $\sim 70 \%$ heteroplasmy in blood) in a patient classified as sporadic. Although the mother of the latter also carried the mutation at a similar level of heteroplasmy in blood, her hearing was apparently normal, as was her brother's. The 7472insC patient had progressive hearing loss from age 5 years. The possible involvement of aminoglycosides in the A1555G families was unclear, but both showed unaffected as well as affected members, the latter having severe to profound, progressive deafness with a downsloping audiogram, and age of onset in the second or third decade. The A3243G family had several members affected by moderate to severe deafness, with age of onset between 10 and 30 years, but with no reports of MELAS or other symptoms. The mutation is known to be under-represented in blood, but heteroplasmy could not be ascertained in other tissues, for ethical reasons. All maternally related individuals in the A7445G family (of Ukrainian origin) had a moderate to severe hearing loss, which began between the ages of 5 and 30. The T7510C family also showed a moderate to severe hearing loss, mostly at the higher frequencies, although the age of onset varied widely, from 1 year to midadulthood.

Four instances of other mtDNA polymorphisms came to light, which might play a role in the phenotypes observed. The novel polymorphism A5568G (tRNA ${ }^{\text {Trp }}$ ) was found in a multigenerational family with hearing impairment that was found subsequently also to have hypopigmentation, apparently transmitted only through females. Mutations in nuclear genes PAX3 and MITF, which have previously been associated with such a phenotype, were excluded. The mutation was absent from $>150$ controls and alters to $\mathrm{G}-\mathrm{U}$ a Watson-Crick base-paring in the T-stem of the tRNA, which is conserved across all vertebrates, suggesting that it may be pathogenic. Another individual with adult-onset, progressive hearing impairment, plus maternal family history, was found to harbour the novel polymorphism G5773A (tRNA ${ }^{\mathrm{Cys}}$ ). This was absent from 143 controls, but the affected nucleotide is not highly conserved, and its functional significance is thus unclear.

A7456G (T-stem of tRNA ${ }^{\text {Ser(UCN)})}$ was found in a single patient with childhood-onset hearing impairment, who subsequently was found also to have diabetes mellitus and colour blindness, but no family history. The mutation was absent from over 150 controls, but strengthens rather than abolishes a base-pairing interaction; therefore, its significance is unpredictable. Finally, the T4336C polymorphism, previously suggested to be a risk factor in Alzheimer's and Parkinson's diseases, ${ }^{34}$ as well as having been reported in families with matrilineal hearing impairment in Finland, ${ }^{35}$ was found in two families, one of which also carried T7510C. While this may be a harmless polymorphism, having been found in $0.7 \%$ of US controls ${ }^{36}$ and in $2.2 \%$ of UK controls, ${ }^{37}$ the possibility that it might act pathogenically in concert with other mitochondrial or nuclear sequence variants remains open.

\section{Causative mtDNA mutations in patients from southern Italy}

Similar analyses were carried out on the patient DNAs from southern Italy. Two cases of A1555G (out of 128 successfully screened), two of A7445G plus one of 7472insC (out of 115 from which tRNA ${ }^{\text {Ser(UCN) }}$ was sequenced) came to light. No cases of A3243G or any other previously reported tRNA $^{\text {Leu(UUR) }}$ mutation were found, among 110 individuals from whom this gene could be sequenced. No other polymorphisms of $\mathrm{tRNA}^{\mathrm{Ser}(\mathrm{UCN})}$ were found. The two cases of A1555G were both familial, occurred on different haplogroup backgrounds, and were homoplasmic. No information on aminoglycoside use by these patients was available. The two cases of A7445G also occurred as homoplasmic mutations on different haplogroup backgrounds ( $\mathrm{T}$ and $\mathrm{J}$ ). The mother of one of the patients was also reported to have postlingual hearing loss and was found to be homoplasmic for the mutation. The patient with 7472insC was initially found to have only approximately $10 \%$ heteroplasmy in blood for the mutation, based on direct sequencing. Although it has not previously been

Table 1 Causative mtDNA mutations found among patients with nonsyndromic hearing impairment

\begin{tabular}{lccc}
\hline Patient group & UK (postlingual) & S. Italy (postlingual) & Finland (ARHI) \\
\hline A1555G & $2 / 80$ & $2 / 128$ & $0 / 227$ \\
A3243G & $1 / 80$ & $0 / 110$ & $0 / 221$ \\
A7445G & $1 / 80$ & $2 / 115$ & $0 / 313$ \\
7472insC & $1 / 80$ & $1 / 115$ & $0 / 313$ \\
T7510C & $1 / 80$ & $0 / 115$ & $0 / 313$ \\
T7511C & $0 / 80$ & $0 / 115$ & $0 / 313$ \\
T7512C & $0 / 80$ & $4.2 \%$ & $0 / 313$ \\
Total frequency (\%) & $7.5 \%$ & $0 \%$ & \\
Other possible mutations & A5568, G5773A, A7456G, T4336C & T3197C, 3159insT, G7521A & ,
\end{tabular}

${ }^{a}$ Heteroplasmic wherever detected.

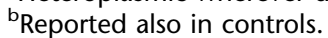


found in controls, and might also be systematically underrepresented in blood, we checked for it in other tissues, using a quantitative, primer extension approach. Hair roots from the patient carried $42 \%$ mutant mtDNA, whereas lymphocytes, urine and cultured fibroblasts showed much lower heteroplasmy levels (15, 11 and 11\%, respectively). Since other studies have shown the mutation to be clearly pathogenic, with the most severely affected individuals showing, in addition to hearing impairment, widespread neurological disease including ataxia and myopathy, we infer that the patient's moderate, adult-onset hearing loss is most likely due to variable tissue heteroplasmy for the mutation.

No novel polymorphisms that are strong candidates for pathogenicity were found among the southern Italian patients. T3197C, found in four patients from two different haplogroups, appears to be a rare polymorphism. The same probably applies to 3159insT, which was found in three patients, two of whom were verified as haplogroup U1. G7521A is potentially more interesting, since it may affect the structure of both tRNA ${ }^{\text {Asp }}$ and pre-tRNA ${ }^{\text {Ser(UCN) }}$ and, to our knowledge, has previously been reported only as a rare polymorphism in nonEuropean haplogroups. We found it in three patients, one of whom was from haplogroup L2a, the others both haplogroup J1a and carrying the same D-loop polymorphisms, although they were not known to have been relatives.

\section{Haplogroup analysis of hearing-impaired patients from UK and southern Italy}

To check whether there was any distortion in the representation of the different mtDNA haplogroups among the patients studied, in comparison with population controls, we analysed informative sites in the mitochondrial genome using a combination of PCR, direct sequencing and primer extension (SNP analysis). Haplogroup assignments were based on the scheme proposed by Macaulay and Richards ${ }^{24,32,33}$ (see Materials and methods and legend to Figure 1). Samples that gave incomplete or uninterpretable results, or where no DNA was available, were excluded. Since total sample numbers were small, we analysed and present the data (Figure 1) in terms of the major European haplogroup clusters.

We were able to define the haplogroup cluster for 123 of the Italian samples, and for 59 of those from the UK. Controls have been previously analysed from both populations, but we co-analysed 149 controls from the UK, as well as 182 from southern Italy (see below), to control for any possible systematic bias in the way we conducted the analysis. An excess of haplogroup cluster HV was evident in both groups of patients compared with population controls, although it was more modest among the Italians. Among the 59 patients from the UK, the excess of patients from haplogroup cluster HV (62 versus $45 \%$ in controls) was reflected by a corresponding deficit of those from haplogroup cluster UK (16 versus 26\% in controls). However, because of low sample numbers, these differences are not statistically significant, based on $\chi^{2}$ testing.

Previous analysis of southern Italian controls ${ }^{30}$ analysed and stratified the data in a slightly different way than in our analysis. RFLPs were used diagnostically, and individuals from the $\mathrm{HV}$ haplogroup cluster who were neither $\mathrm{H}$ nor $\mathrm{V}$, but representatives of earlier branches of the cluster more commonly found in the near East than in Europe, ${ }^{33}$ were presumably placed in the 'other' category', together with those from African or East Eurasian haplogroups, or rarer haplogroups from the $\mathrm{N}$ cluster. The apparently significant excess of haplogroup cluster $\mathrm{HV}$ among the patients we analysed, over the previously published population controls (54 versus $41 \%, P<0.05$ ), is mainly compensated by a drop in this heterogeneous 'other' category. To determine whether it was purely an artefact of the different way the previous authors had categorized haplogroup affiliations, we tested 182 controls from the southern Italian population for membership of the HV haplogroup cluster, using the diagnostic site at np 11719 to generate the most inclusive definition thereof. We found $82 / 182$, that is, $45 \%$ of southern Italian controls, to derive from haplogroup cluster $\mathrm{HV}$, and used this to adjust the published figures by re-assigning a low proportion of 'other' haplogroups to cluster HV. After this adjustment, the $P$-value extrapolated from $\chi^{2}$ testing rises to slightly above 0.05 , and thus the excess of haplogroup cluster HV would no longer be considered significant.

\section{mtDNA mutations in patients with age-related hearing impairment}

Although the patients collected both in Italy and in the UK included some with ages of onset in mid- to late adulthood, all of the cases of causative mtDNA mutations that came to light showed age of onset in childhood or early adult life. This prompted the question as to whether the same mutations are found among the even larger group of patients who first present with hearing loss in late adulthood (ie with ARHI), which affects $\sim 20 \%$ of the population already between 51 and 60 years of age. ${ }^{22}$ To address this issue, focusing on the more clinically severe cases of ARHI, we collected blood DNAs from a series of adult patients attending the ENT clinic of a regional hospital in Finland for first fitting of a hearing aid. The patients typically ranged from 60 to 90 years of age. We tested A1555G and A3243G by primer extension (SNP analysis), in order to maximize the chances of detecting low-level heteroplasmy, and sequenced the genes for tRNA $^{\text {Leu(UUR) }}$ and tRNA ${ }^{\text {Ser(UCN) }}$ together with their flanking regions. No instances of any of the known, causative mtDNA mutations were found at a detectable level of heteroplasmy, among the following numbers of patients 
screened: 0/227 for A1555G, 0/313 for A7445G, 7472insC or any other mutations in tRNA ${ }^{\operatorname{Ser}(\mathrm{UCN})}$ and $0 / 221$ for A3243G or any other mutations in tRNA ${ }^{\text {Leu(UUR) }}$. We also analysed haplogroup affiliations in these patients, for evidence of the same distortion in favour of haplogroup cluster HV as seen in patients from the UK or southern Italy with postlingual hearing impairment. The results for 172 patients (median age 76 years, mean 73.6 ), which we could definitively assign to a specific haplogroup cluster (Figure 1), indicate instead a slight but statistically nonsignificant deficiency of haplogroup cluster HV (46\%) compared with published data ${ }^{31}$ for the relevant control population $(55 \%)$ or unpublished data collected by others (A Sajantila, personal communication). We also found no significant deviations from population controls of haplogroup cluster frequencies among UK patients with late adult-onset hearing loss (data not shown).

\section{Discussion \\ Pathogenic mtDNA mutations in patients with postlingual, nonsyndromic hearing impairment}

Previous authors have found the major pathogenic mutations A1555G, A3243G, A7445G, 7472insC and T7510C both in individual maternal pedigrees with early-onset, progressive hearing impairment and in screens of hearingimpaired patients classified in various ways. This study is the first one, however, which has looked systematically for all of these mutations in patients with postlingual hearing impairment, from two different European populations. Despite the slightly different criteria used for patient recruitment, the results are strikingly concordant: in both groups, approximately $5 \%$ of patients had one of the previously identified, pathogenic mtDNA mutations $(7.5 \%$ of UK patients, $4.2 \%$ of Italian patients), which rises to almost $10 \%$ of clearly familial cases in both populations. The only positives among nonfamilial cases were the two instances of 7472insC, both heteroplasmic. Patients with pathogenic mtDNA mutations had ages of onset before 30, with several in childhood, and typically showed progression of the disorder.

However, we would argue that restricting too narrowly the criteria for the identification of patients with mitochondrial hearing impairment will inevitably lead to some such individuals being missed. Most modern-day European families are small and dispersed. Moreover, environmental factors such as aminoglycosides are known to play a role in expression of the clinical phenotype, at least for A1555G. Verifying progression of the phenotype requires that the patient be tested using the same protocol at different times, which is not necessarily feasible or likely.

Although there is currently no therapy available for mitochondrial hearing impairment, this will not necessarily be so in the future. Future therapeutic developments may be of value to patients identified in the present, having a progressive disorder. In addition, genetic counselling will be appropriate. We therefore recommend that all patients with postlingual hearing impairment be routinely screened for A1555G, A3243G, and for all known causative mutations affecting tRNA ${ }^{\operatorname{Ser}(\mathrm{UCN})}$, certainly including those that were found in our survey. Because of the tendency for hearing-impaired individuals to marry each other, the presence of a hearing disorder in the father or paternal relatives should not be taken as an exclusion criterion. Since A1555G has also been reported in patients with prelingual deafness, ${ }^{1,21}$ we suggest that screening might usefully be applied routinely to all individuals with unexplained hearing impairment, with age of onset in infancy, childhood or early adulthood.

\section{Heteroplasmy for 7472insC}

The 7472insC mutation was previously reported in patients with nonsyndromic hearing impairment, ${ }^{5}$ as well as in other patients with a wider neurological syndrome, ${ }^{4}$ in both cases at a high level of heteroplasmy, typically $>85 \%$. In this study, we found two cases of the mutation at a lower level of heteroplasmy (in one case $\sim 70 \%$ in blood, the other $42 \%$ in hair roots and even less in blood), but with sensorineural hearing impairment as the only feature. Some unaffected relatives showed a similar level of heteroplasmy, raising the possibility that other mutations might contribute to the expression of the mutant phenotype in such cases. However, heteroplasmy for the mutation should clearly be considered and screened for in patients with hearing impairment.

\section{Frequency of A1555G among patients with} progressive, postlingual hearing impairment

Our findings are in line with most other reports of the frequency of A1555G among Europeans with familial hearing impairment, ${ }^{21,38,39}$ but strikingly different from studies conducted in Spain, where A1555G has been found at a much higher frequency, up to $\sim 25 \%$ of individuals with late-onset, familial, progressive hearing impairment. ${ }^{40-42}$ Since such studies have been carried out in more than one laboratory, it is unlikely that they are due to any systematic bias in the collection of subjects. The type of hearing disorder resulting from A7445G, T7510C or 7472 ins $C$ is quite similar to that in A1555G families, hence it is also unlikely that our failure to identify more A1555G patients in Italy or the UK is because of a systematic bias in our own collection of patients, which did include patients with tRNA ${ }^{\mathrm{Ser}(\mathrm{UCN})}$ mutations. Note that $70 \%$ of UK cases and $40 \%$ of Italian cases studied were familial, so that we would have expected at least $10-15 \%$ of them to test positive for $\mathrm{A} 1555 \mathrm{G}$, if the occurrence of the mutation as a cause of hearing impairment was comparable to that in Spain.

This leaves two possible explanations for the discordance: environmental factors, such as unrecorded aminoglycoside 
use, A1555G being a known ototoxic risk factor, ${ }^{43}$ or else other genetic factors, that is, multiple founder effects or systematic selection (A1555G being found in different mtDNA haplogroups ${ }^{41}$ ). An unbiased evaluation of the population prevalence of A1555G in different European countries can perhaps address this issue. Nuclear genotypic differences also cannot be ruled out, but would, as far as we are aware, be unprecedented in such large and heterogeneous populations.

\section{Other mtDNA polymorphisms and postlingual hearing impairment}

The pathological significance of novel, rare polymorphisms in single pedigrees is impossible to assess. While phylogenetic conservation or structural effects (for tRNA gene polymorphisms) are suggestive, they are not definitive. Ultra-rare or private polymorphisms need not be deleterious, whereas low-penetrance mutations are, by definition, found also among controls. Thus, A5568G might contribute to hearing impairment, whereas G7521A might not, or vice versa. The effect of mitochondrial (or, indeed, nuclear) genetic background may determine the issue in a given case. An increased number of rare polymorphisms has been reported among Finnish patients with sensorineural hearing impairment, compared with controls, consistent with at least some of them being mildly deleterious mutations contributing collectively to the phenotype. ${ }^{44}$ The mtDNA polymorphisms reported here should be considered as possible candidates for involvement in hearing disorders, but there is at this time no compelling evidence to support any.

\section{Mitochondrial haplogroup affiliations and hearing impairment}

Previous authors have suggested mitochondrial haplogroup associations with various disorders ${ }^{25-29}$ or with longevity, ${ }^{30,31}$ and as a possible modifier of mitochondrial disease, ${ }^{45}$ although contrary data also exist. ${ }^{46}$ Our data suggest a possible distortion towards haplogroup cluster HV among patients with early-onset but postlingual hearing impairment, at least compared with population controls or patients with ARHI, although of borderline statistical significance. Previous associations with longevity $^{31}$ suggest that tightly age-matched controls might be needed to judge the issue definitively. One possible explanation for such a bias would be that other, as yet unidentified pathological mutations may have arisen on the HV background.

\section{Conclusion}

We found that $5 \%$ or more of cases of postlingual, nonsyndromic hearing impairment in two geographically distant regions of Europe are attributable to known mtDNA mutations. Collectively they thus represent one of most frequent genetic causes of hearing impairment, and therefore should be routinely screened for, especially as their identification has quite different genetic counselling implications from nuclear gene mutations. The possible deviation of mtDNA haplogroup frequencies among these patients suggests that mtDNA genotype might contribute to hearing impairment in an even larger proportion of cases. Further studies of large numbers of such patients is needed to evaluate the significance of possible haplogroup deviations.

\section{Acknowledgements}

This work was supported by EU Concerted Action GENDEAF (QLG1CT-2001-01429), the Academy of Finland, Tampere University Medical Research Fund, the Royal National Institute for the Deaf, Fondazione Telethon-Italy (Grant no. GGP030039), Fondazione Pierfranco \& Luisa Mariani (Ricerca 2000), Ricerca Finalizzata Ministero della Salute RF-2002/158 and Fondazione Cariplo (Ricerca 2002). We thank Johanna Lauttanen and Minna Kokkonen for technical assistance, Graham Taylor and Teresa Palladino for archiving of DNA samples, and three anonymous reviewers for very helpful comments.

\section{References}

1 Prezant TR, Agapian JV, Bohlman MC et al: Mitochondrial ribosomal RNA mutation associated with both antibioticinduced and non-syndromic deafness. Nat Genet 1993; 4: 289-294.

2 Reid FM, Vernham GA, Jacobs HT: A novel mitochondrial point mutation in a maternal pedigree with sensorineural deafness. Hum Mutat 1994; 3: 243-247.

3 Fischel-Ghodsian N, Prezant TR, Fournier P, Stewart IA, Maw M: Mitochondrial mutation associated with nonsyndromic deafness. Am J Otolaryngol 1995; 16: 403-408.

4 Tiranti V, Chariot P, Carella F et al: Maternally inherited hearing loss, ataxia and myoclonus associated with a novel point mutation in mitochondrial tRNASer(UCN) gene. Hum Mol Genet 1995; 4: 1421-1427.

5 Verhoeven K, Ensink RJ, Tiranti $\mathrm{V}$ et al: Hearing impairment and neurological dysfunction associated with a mutation in the mitochondrial tRNASer(UCN) gene. Eur J Hum Genet 1999; 7: $45-51$.

6 van den Ouweland JM, Lemkes HH, Ruitenbeek W et al: Mutation in mitochondrial tRNA(Leu)(UUR) gene in a large pedigree with maternally transmitted type II diabetes mellitus and deafness. Nat Genet 1992; 1: 368-371.

7 Majamaa K, Moilanen JS, Uimonen S et al: Epidemiology of A3243G, the mutation for mitochondrial encephalomyopathy, lactic acidosis, and strokelike episodes: prevalence of the mutation in an adult population. Am J Hum Genet 1998; 63: $447-454$.

8 Reid FM, Rovio A, Holt IJ, Jacobs HT: Molecular phenotype of a human lymphoblastoid cell-line homoplasmic for the np 7445 deafness-associated mitochondrial mutation. Hum Mol Genet 1997; 6: 443-449.

9 El Meziane A, Lehtinen SK, Hance N et al: A tRNA suppressor mutation in human mitochondria. Nat Genet 1998; 18: 350-353.

10 Guan MX, Enriquez JA, Fischel-Ghodsian N et al: The deafnessassociated mitochondrial DNA mutation at position 7445, which affects tRNASer(UCN) precursor processing, has long-range effects on NADH dehydrogenase subunit ND6 gene expression. Mol Cell Biol 1998; 18: 5868-5879.

11 Guan MX, Fischel-Ghodsian N, Attardi G: Nuclear background determines biochemical phenotype in the deafness-associated 
mitochondrial 12S rRNA mutation. Hum Mol Genet 2001; 10: $573-580$

12 Chomyn A, Enriquez JA, Micol V, Fernandez-Silva P, Attardi G: The mitochondrial myopathy, encephalopathy, lactic acidosis, and stroke-like episode syndrome-associated human mitochondrial tRNALeu(UUR) mutation causes aminoacylation deficiency and concomitant reduced association of mRNA with ribosomes. J Biol Chem 2000; 275: 19198-19209.

13 Toompuu M, Yasukawa T, Suzuki T et al: The 7472insC mitochondrial DNA mutation impairs the synthesis and extent of aminoacylation of tRNASer(UCN) but not its structure or rate of turnover. J Biol Chem 2002; 277: 22240-22250.

14 Hutchin TP, Parker MJ, Young ID et al: A novel mutation in the mitochondrial tRNA(Ser(UCN)) gene in a family with nonsyndromic sensorineural hearing impairment. J Med Genet 2000; 37: $692-694$.

15 del Castillo FJ, Villamar M, Moreno-Pelayo MA et al: Maternally inherited non-syndromic hearing impairment in a Spanish family with the $7510 \mathrm{~T}>\mathrm{C}$ mutation in the mitochondrial tRNA(Ser(UCN)) gene. J Med Genet 2002; 39: e82.

16 Sue CM, Tanji K, Hadjigeorgiou G et al: Maternally inherited hearing loss in a large kindred with a novel T7511C mutation in the mitochondrial DNA tRNA(Ser(UCN)) gene. Neurology 1999; 52: $1905-1908$

17 Chapiro E, Feldmann D, Denoyelle F et al: Two large French pedigrees with non syndromic sensorineural deafness and the mitochondrial DNA T7511C mutation: evidence for a modulatory factor. Eur J Hum Genet 2002; 10: 851-856.

18 Jaksch M, Klopstock T, Kurlemann G et al: Progressive myoclonus epilepsy and mitochondrial myopathy associated with mutations in the tRNA(Ser(UCN)) gene. Ann Neurol 1998; 44: 635-640.

19 Zhao H, Li R, Wang Q et al: Maternally inherited aminoglycosideinduced and nonsyndromic deafness is associated with the novel C1494T mutation in the mitochondrial 12S rRNA gene in a large Chinese family. Am J Hum Genet 2004; 74: 139-152.

20 Hutchin TP, Thompson KR, Parker M, Newton V, Bitner-Glindzicz M, Mueller RF: Prevalence of mitochondrial DNA mutations in childhood/congenital onset non-syndromal sensorineural hearing impairment. J Med Genet 2001; 38: 229-231.

21 Tekin M, Duman T, Bogoclu G et al: Frequency of mtDNA A1555G and A7445G mutations among children with prelingual deafness in Turkey. Eur J Pediatr 2003; 162: 154-158.

22 Davis AC: The prevalence of hearing impairment and reported hearing disability among adults in Great Britain. Int J Epidemiol 1989; 18: 911-917.

23 Fortnum HM, Summerfield AQ, Marshall DH, Davis AC, Bamford JM: Prevalence of permanent childhood hearing impairment in the United Kingdom and implications for universal neonatal hearing screening: questionnaire based ascertainment study. $B M J$ 2001; 323: 536-540.

24 Richards M, Macaulay V, Torroni A, Bandelt HJ: In search of geographical patterns in European mitochondrial DNA. Am J Hum Genet 2002; 71: 1168-1174.

25 Ruiz-Pesini E, Lapena AC, Diez-Sanchez C et al: Human mtDNA haplogroups associated with high or reduced spermatozoa motility. Am J Hum Genet 2000; 67: 682-696.

26 van der Walt JM, Nicodemus KK, Martin ER et al: Mitochondrial polymorphisms significantly reduce the risk of Parkinson disease. Am J Hum Genet 2003; 72: 804-811.

27 Chinnery PF, Taylor GA, Howell N et al: Mitochondrial DNA haplogroups and susceptibility to $\mathrm{AD}$ and dementia with Lewy bodies. Neurology 2000; 55: 302-304.
28 Carrieri G, Bonafe $M$, De Luca $M$ et al: Mitochondrial DNA haplogroups and APOE4 allele are non-independent variables in sporadic Alzheimer's disease. Hum Genet 2001; 108: 194-198.

29 Kalman B, Li S, Chatterjee D et al: Large scale screening of the mitochondrial DNA reveals no pathogenic mutations but a haplotype associated with multiple sclerosis in Caucasians. Acta Neurol Scand 1999; 99: 16-25.

30 De Benedictis G, Rose G, Carrieri G et al: Mitochondrial DNA inherited variants are associated with successful aging and longevity in humans. FASEB J 1999; 13: 1532-1536.

31 Niemi AK, Hervonen A, Hurme M, Karhunen PJ, Jylha M, Majamaa K: Mitochondrial DNA polymorphisms associated with longevity in a Finnish population. Hum Genet 2003; 112: 29-33.

32 Macaulay V, Richards M, Hickey E et al: The emerging tree of West Eurasian mtDNAs: a synthesis of control-region sequences and RFLPs. Am J Hum Genet 1999; 64: 232-249.

33 Richards M, Macaulay V, Hickey E et al: Tracing European founder lineages in the near Eastern mtDNA pool. Am J Hum Genet 2000; 67: $1251-1276$

34 Wallace DC: Mitochondrial DNA sequence variation in human evolution and disease. Proc Natl Acad Sci USA 1994; 91: 8739-8746.

35 Finnila S, Autere J, Lehtovirta M: Increased risk of sensorineural hearing loss and migraine in patients with a rare mitochondrial DNA variant 4336A > G in tRNAGln. J Med Genet 2001; 38: 400-405.

36 Shoffner JM, Brown MD, Torroni A et al: Mitochondrial DNA variants observed in Alzheimer disease and Parkinson disease patients. Genomics 1993; 17: 171-184

37 Hutchin TP, Heath PR, Pearson RC, Sinclair AJ: Mitochondrial DNA mutations in Alzheimer's disease. Biochem Biophys Res Commun 1997; 241: 221-225.

38 Kupka S, Toth T, Wrobel M et al: Mutation A1555G in the 12S rRNA gene and its epidemiological importance in German, Hungarian, and Polish patients. Hum Mutat 2002; 19: 308-309.

39 Ostergaard E, Montserrat-Sentis B, Gronskov K, Brondum-Nielsen $\mathrm{K}$ : The A1555G mtDNA mutation in Danish hearing-impaired patients: frequency and clinical signs. Clin Genet 2002; 62: 303-305.

40 Estivill X, Govea N, Barcelo E et al: Familial progressive sensorineural deafness is mainly due to the mtDNA A1555G mutation and is enhanced by treatment of aminoglycosides. Am J Hum Genet 1998; 62: 27-35.

41 Torroni A, Cruciani F, Rengo C et al: The A1555G mutation in the $12 S$ rRNA gene of human mtDNA: recurrent origins and founder events in families affected by sensorineural deafness. Am J Hum Genet 1999; 65: 1349-1358.

42 Gallo-Teran J, Morales-Angulo C, del Castillo I, Moreno-Pelayo MA, Mazon A, Moreno F: Familial susceptibility to aminoglycoside ototoxicity due to the A1555G mutation in the mitochondrial DNA. Med Clin (Barc) 2003; 121: 216-218.

43 Fischel-Ghodsian N, Prezant TR, Chaltraw WE et al: Mitochondrial gene mutation is a significant predisposing factor in aminoglycoside ototoxicity. Am J Otolaryngol 1997; 18: 173-178.

44 Lehtonen MS, Moilanen JS, Majamaa K: Increased variation in mtDNA in patients with familial sensorineural hearing impairment. Hum Genet 2003; 113: 220-227.

45 Crimi M, Del Bo R, Galbiati S et al: Mitochondrial A12308G polymorphism affects clinical features in patients with single mtDNA macrodeletion. Eur J Hum Genet 2003; 11: 896-898.

46 Torroni A, Campos Y, Rengo C et al: Mitochondrial DNA haplogroups do not play a role in the variable phenotypic presentation of the A3243G mutation. Am J Hum Genet 2003; 72: $1005-1012$. 